\title{
LA POLÍTICA SOCIAL Y ECONÓMICA DEL GOBIERNO DEL FRENTE AMPLIO EN URUGUAY (2005-2016)
}

\author{
Luis Miguel Uharte \\ Universidad del País Vasco - Euskal Herriko Unibertsitatea
}

https://doi.org/10.33676/EMUI_nomads.56.06

\begin{abstract}
Resumen: El siguiente artículo se propone realizar un análisis de la política social y económica del gobierno del Frente Amplio (FA) en Uruguay, de 2005 hasta 2016, señalando los cambios más relevantes y las contradicciones más destacables en estos dos ámbitos. El balance se realiza a partir de una comparación con la situación existente durante el anterior periodo neoliberal. Se concluye que el cambio ha sido real pero moderado y se ha evidenciado sobre todo en algunas reformas sociales (salud, laboral y tributaria). En el área económica los resultados macroeconómicos han sido satisfactorios, pero el modelo económico no se revertido sino todo lo contrario, se ha acentuado en sus problemas tradicionales (primarización, concentración y extranjerización de propiedad, contaminación ambiental). La metodología combina la revisión bibliográfica con entrevistas semi-estructuradas realizadas durante el año 2017.
\end{abstract}

Palabras clave: Frente Amplio, cambio moderado, reformas sociales, política económica.

\section{The social and economic policy of the government of the Broad Front in Uruguay (2005-2016)}

Abstract: This paper aims to analyze the social and economic policy of the government of the 'Broad Front' in Uruguay, from 2005 to 2016, identifying the most relevant changes and the most notable contradictions in these two areas. We have made a comparison with the situation existing during the previous neoliberal period. We concluded that the change has been real but moderate and has been evident above all in some social reforms (health, labor and tax). In the economic area, the macroeconomic results have been satisfactory, but the economic model has not changed; it has worsened in its traditional problems (primarization, concentration and foreign ownership of property, environmental pollution). The methodology combines bibliographic review with semi-structured interviews conducted during the year 2017.

Keywords: Broad Front, moderate change, social reforms, economic policy

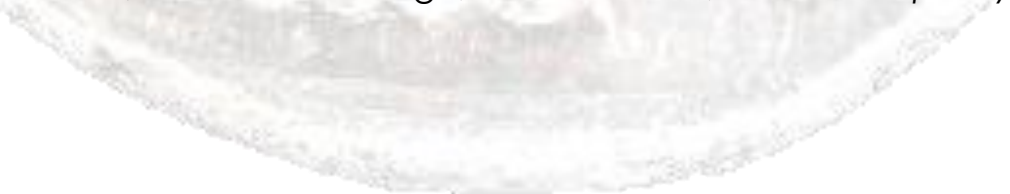

La victoria en las elecciones del Frente Amplio (FA) en octubre de 2004, supuso un cambio político de enorme trascendencia en Uruguay, ya que por primera vez en la historia no ganaba uno de los dos partidos tradicionales (ni el Colorado ni el Nacional) y además resultaba triunfante un partido de izquierdas (Caetano, 2016a: 35; Arocena, 2005: 152; Lorenzoni y Pérez, 2013: 82). 
La crisis económica fue un detonante importante ya que el deterioro de las condiciones de vida de una gran parte de la población llevó a un rechazo masivo al neoliberalismo y a los dos partidos que lo sustentaban (Arocena, 2005: 152). A partir de la asunción de gobierno en marzo de 2005, el FA impulso una agenda de cambios, en el área social y económica. A continuación vamos a realizar un balance de estas dos áreas, intentando identificar las transformaciones más importantes y las limitaciones más evidentes.

\section{Política social}

\section{Políticas de salud y educación}

Uno de los íconos del cambio en política social de los gobiernos del FA ha sido la reforma de la salud. El modelo de salud a lo largo del siglo XX fue segmentado según el estrato social ya que había 3 subsistemas: "el privado con fines de lucro destinado a la atención de sectores socioeconómicos altos y medio-altos; un subsistema también privado, en muchos casos sin fines de lucro y mutual-principalmente a través de las Instituciones de Asistencia Médica Colectiva (IAMC)- que brindó cobertura a los trabajadores formales y a sus familias a partir de las contribuciones a la seguridad social y un subsector público cuyo cometido fue el de brindar asistencia a los sectores vulnerables y aquellos pertenecientes al sector informal de la economía". Traducido en porcentajes, en 2005 el $51 \%$ se atendía en centros públicos, un $43 \%$ en algún IAMC y en privados un 2\% (Antia et al, 2013: 182-3).

La reforma del Sistema de Salud se impulsó en el primer año de mandato, en el 2005. El objetivo era "avanzar hacia una cobertura universal en salud" y para ello se creó el Fondo Nacional de Salud (FONASA) (MIDES, 2014: 56). El aumento de la cobertura se produce porque desaparece el DISSE (Dirección de Seguros Sociales por Enfermedad), "que sólo aseguraba a asalariados/s del sector privado", y lo sustituye el citado FONASA, "que asegura a todas las personas asalariadas, tanto del sector público como del privado". La cobertura aumenta de manera exponencial ya que se pasa de cubrir a 586.000 personas en el 2005 a 2.600 .000 en el 2107 , alrededor de un $80 \%$ de la población (Olesker, 2017).

La reforma también dio la opción a todas las usuarias y usuarios de elegir entre ASSE (Administración de Servicios de Salud del Estado), es decir, el prestador estatal de salud pública, y las IAMC, es decir, las mutuales. La consecuencia fue un trasvase importante de un sector de la población de ASSE hacia las mutuales. El prestador público pasó de cubrir al $50,8 \%$ del total de la población al $34 \%$, mientras que las IAMC "crecieron casi un 15\%", llegando al "58\% de la población". La 
consecuencia, por tanto, ha sido una "fuga" hacia las mutuales (Antia et al, 2013: 184).

De cualquier manera, hay una serie de indicadores de salud que han mejorado sustancialmente. Por un lado, el gasto público de salud ha aumentado acercándose cada vez más al mutual. En 2004 ASSE gastaba por persona un tercio de lo que gastaban las mutuales, mientras que en 2012 se acercó a un 75\% (Olesker, 2014a:21). Respecto al PIB, el gasto de salud se habría casi duplicado, subiendo del 3,2\% en 2004 al 6,1\% en 2012. Un último indicador relevante de salud es la mortalidad infantil, que se ha reducido del 14 al 7 por mil (Olesker, 2017).

En el área de educación no ha habido una reforma de gran calado como en salud. De todas formas, hay que destacar el incremento sustancial del gasto público educativo y las consecuencias derivadas de esto en relación al aumento salarial de docentes y a la mejora de la cobertura para los sectores más excluidos en todos los tramos (primaria, secundaria y terciaria).

El aumento del gasto público en educación es significativo, ya que se pasa de un 3,2\% en 2004 a un 4,7\% en 2013 (De Armas, 2017: 191). Esto permitió un aumento del salario docente "entre 70 y $80 \%$ " de 2005 a 2014 (Olesker, 2014a: 19). A su vez, "la brecha entre los proveedores estatales y los privados (medida a través del costo por estudiante) se redujo en la educación pre-terciaria y universitaria". En el tramo preuniversitario "la ratio entre el costo por estudiante en el sistema público y el correspondiente al sistema privado aumentó de $59 \%$ en 2006 a $87 \%$ en 2012 , en tanto en la educación universitaria pasó de $40 \%$ a 73\%" (De Armas, 2017: 349).

Este aumento del gasto posibilitó que "muchos hogares excluidos en los años 90 pudieron reincorporar sus hijas e hijos al sistema educativo". En educación inicial (de 0 a 3 años), por ejemplo, se pasó de 21,7\% de cobertura ( 1 de cada cinco niñas/os) a 35,1\% ( 1 de cada 3 ). En este tramo, "las familias del $15 \%$ más pobre de la población duplicaron su asistencia a la educación" (MIDES, 2014: 7).

Hay que destacar también un aumento significativo de la matrícula universitaria, "en particular en el interior del país", con la creación o fortalecimiento de "polos regionales de la Universidad de la República", mejorando el acceso de "segmentos de la población que anteriormente enfrentaban dificultades de acceso" (De Armas, 2017: 349). El alumnado universitario creció un $37 \%$ durante ese periodo (de 107.000 estudiantes a 147.000) y concretamente "la población estudiantil residente en el interior del país creció de 36 mil a 58 mil (un crecimiento de 61\%)" (De Armas, 2017: 200). Delacoste (2017) destaca 
también el avance en formación del profesorado y el consiguiente aumento de la investigación de posgrado.

El tercer gobierno del FA ha planteado como una de las "grandes reformas sociales de su nuevo mandato" la creación del Sistema Nacional de Cuidados (SNC) (Pereira, 2017). Tabaré Vázquez "catalogó al SNC -destinado a la atención de las necesidades de cuidado de las personas dependientes, en particular ancianos, personas con discapacidad y niños/as pequeños-como el "buque insignia" de su segunda gestión" (Pérez y Piñeiro, 2016: 349).

Las reformas en salud y educación han ido de la mano del aumento del gasto social, muy superior al de la época neoliberal. Según el MIDES del 2005 al 2012 el gasto público social (GPS) creció un $87 \%$, lo que supone "un crecimiento promedio anual de $8,4 \%$, mientras que en el periodo 1989 a 2004 fue el 4,4\%". Creció "41 puntos por encima del PIB (que creció $46,6 \%$ en ese mismo período) y 35 puntos por encima del gasto público total (GPT), (que creció 52,2\%)". Esto evidencia, según el MIDES, "la prioridad política en el incremento del Gasto Público Social". Como porcentaje del PIB pasó del 19,5\% de 2005 al 25,2\% en 2014 y en relación con el total del gasto público pasó de representar en 2004 el $60,9 \%$ del GPT a ser en 2012 el 75,4\% (MIDES, 2014: 9).

\section{Políticas laborales y pensiones}

Los avances en el área laboral son evidentes, ya que es reconocido incluso por voces muy críticas con el gobierno (Elias, 2010; Mañan, 2013; Notaro, 2015). Las principales medidas impulsadas por los gobiernos del Frente Amplio se pueden agrupar en 3 bloques: por un lado, la reactivación de los Consejos de Salarios y de la negociación colectiva; por otro lado, el estímulo a la formalización del empleo; finalmente, las leyes de protección de la acción sindical.

La reactivación de los Consejos de Salarios fue una de las primeras medidas del FA. Los Consejos de Salarios surgieron en los años 40 y fueron suprimidos poco antes de la dictadura, permaneciendo desactivados de facto durante el neoliberalismo, por lo que su reactivación en 2005 es un logro indudable. Además, a partir de entonces amplían su intervención "a tres categorías de trabajadores históricamente no contempladas en esas instancias": el sector público, el sector rural y el sector doméstico (Midaglia, 2018: 8). Mañan (2013: 43) agrega que "otro avance es la ley de negociación colectiva, tanto para el sector público como para el privado". En una década (20052014), según Carracedo y Senatore (2016: 33), "la cobertura de la negociación colectiva pasó del $20 \%$ a prácticamente el $90 \%$ de los asalariados". 
Las políticas para aumentar la formalización del empleo son otro de los ámbitos reseñables. Las medidas más relevantes en este campo fueron el aumento de "las instancias de fiscalización" y la reglamentación de las "condiciones de trabajo de aquellas categorías laborales en las que tiene fuerte incidencia la informalidad (trabajadoras domésticas, etc.) (Midaglia, 2018: 10). La "regularización de más de 12.000 trabajadores/as que se encontraban con contratos precarios desde hace mucho tiempo en la administración" (Mañan, 2013: 43) es otra medida destacable.

Los datos estadísticos de mejora de la formalización son contundentes. La informalidad se redujo del $35 \%$ al $25 \%$ entre 2006 y 2016 (INE, 2017, en Midaglia, 2018: 10). La formalización ha supuesto también una ampliación de derechos (aguinaldo, vacaciones pagadas, seguro por enfermedad, derecho a indemnización por despido y a jubilación) (MIDES, 2014: 6).

Durante la primera década de gobierno del FA el número de cotizantes al BPS (Banco de Previsión Social), a través del cual se accede a la Seguridad Social, ha aumentado un $60 \%$, pasando de menos de un millón de afiliados a más de millón y medio (MIDES, 2014: 34).

De todas formas, todavía el mercado de trabajo sigue "mostrando elevados niveles de segmentación", ya que un $80 \%$ de los asalariados informales perciben salarios muy bajos, por debajo del "valor equivalente a dos líneas de pobreza urbana per cápita". A su vez, "entre los trabajadores de bajo nivel de calificación los formales percibían un ingreso $120 \%$ superior al de los informales". En resumen, "aún persisten importantes niveles de dualización" entre formales e informales (Antia et al, 2013: 177-180). Además, el incremento del empleo ha sido fundamentalmente en los servicios, un sector con empleos más precarios que en la industria (Zibechi, 2017; Delacoste, 2017).

El otro campo donde se ha dado un avance sustantivo es en la aprobación de "normas que otorgan mayores garantías a la acción sindical", destacándose sobre todo la "Ley de Fuero Sindical", además de la ya citada reactivación de los Consejos de Salarios. Esto influyó directamente en el aumento de la tasa de sindicalización, ya que se pasó de un $20 \%$ de promedio en los años noventa a un $29 \%$ en 2014. Carracedo y Senatore destacan que esta tasa está por encima de "países como Alemania (25\%), España (16 \%) y Reino Unido (25\%)" (Carracedo y Senatore, 2016: 33).

Las citadas reformas en el ámbito laboral posibilitaron además una mejora sustancial de dos indicadores básicos: el salario real y el empleo. 
En cuanto al salario real, Olesker recuerda que durante los años 90 cayó en términos reales un 50\% (Olesker, 2014a: 11), mientras que de 2004 a 2015 se incrementó un 52\% (Carracedo y Senatore, 2016: 24). El salario mínimo real tuvo un comportamiento aún mejor, ya que durante la primera década del FA ha crecido un $130 \%$ en términos reales (Carracedo y Senatore, 2016: 25). La tasa de desempleo también se ha reducido radicalmente, en un $40 \%$ durante la primera década, pasando del $10,8 \%$ al $6,5 \%$. La reducción fue mayor en mujeres que hombres aunque estas todavía sufren una tasa mayor, un $8 \%$ frente al $5 \%$ de estos (MIDES, 2014: 32-3).

Si en el ámbito laboral los avances son evidentes en materia de pensiones los resultados son muy diferentes. Midaglia recuerda que en los años noventa el sistema de jubilaciones y pensiones había sido parcialmente liberalizado "incorporando al clásico esquema estatal de reparto intergeneracional un pilar de capitalización individual". En ninguno de los 3 programas de gobierno del FA figuraba alguna propuesta para retornar al anterior sistema, aunque sí "se introdujeron un paquete medidas tendientes a flexibilizar las exigencias de acceso a las prestaciones jubilatorias" (reducción de 35 a 30 años el tiempo de cotización y bajada de la edad de jubilación) (Midaglia, 2018: 17).

Para Midaglia (2018: 17), el FA "adoptó una estrategia de acción relativamente conservadora en materia previsional, en el sentido de promocionar cambios de pequeña envergadura" debido a la falta de "una amplia coalición sociopolítica para revisar bajo parámetros alternativos el esquema vigente y el peligro de desatar un nuevo proceso de conflictividad política en el que los grupos corporativos se posicionaran en defensa y aumento de sus beneficios".

Antia et al (2013: 181-2) señalan otras medidas en materia de jubilación: "la creación de un subsidio de asistencia a la vejez para las personas mayores de 64 y menores de 70 años en condiciones económicas críticas", y "el reconocimiento a las mujeres trabajadoras de un año de servicio por cada hijo". Sin embargo, indican también el "elevado nivel de segmentación", ya que los montos de las jubilaciones que perciben las personas que se retiran dentro del régimen de reparto administrado por el BPS son considerablemente más bajos qué los que los que lo hacen a través de alguna de las cajas para-estatales". A su vez, destacan la gran diferencia entre las pensiones contributivas y no contributivas, ya que estas últimas representan el $45 \%$ del monto de las primeras. 


\section{Pobreza y desigualdad}

La reducción drástica de la pobreza y de la pobreza extrema durante la primera década de gobierno es una de las banderas del Frente Amplio en la actualidad. Entre 2005 y 2015 la pobreza se redujo del $32,5 \%$ al $9,7 \%$ y la extrema del 2,2\% al 0,3\% (Midaglia, 2018: 18). Sería el registro más bajo de pobreza de los últimos 30 años según Caetano. Agrega el autor que "si se utiliza la Línea de Pobreza de CEPAL, se puede afirmar que Uruguay alcanzó en 2012 el registro más bajo de la región y, además, el más bajo desde la década del sesenta (Caetano, 2016a: 3940).

Pero a su vez, la reducción de la pobreza sigue lastrada por una tendencia histórica, "su concentración territorial (asociada a procesos de segmentación residencial) y a una "sobrerrepresentación en las generaciones más jóvenes (Caetano, 2016a: 40). El propio MIDES (2014: 19-20) reconoce que "no se ha revertido la correlación negativa preexistente entre la edad de las personas y las posibilidades de encontrarse en situación de pobreza; a menor edad, mayor es la probabilidad de ser pobre". De hecho en 2013 "la pobreza entre los menores de 18 años fue de $21,2 \%$, menos de la mitad que en 2006", pero el doble que el total de la población.

Los balances en torno a la desigualdad son diferentes según los/as autores/as. Por una parte, tenemos a aquellos/as que basándose en el clásico índice de GINI afirman categóricamente que la desigualdad se ha reducido sustancialmente. Olesker (2014a: 28), por ejemplo, utilizando el citado índice de GINI indica que en los años noventa la desigualdad aumento del 0,41 al 0,43 , mientras que durante la primera década de gobierno del FA se ha reducido pasando del 0,45 al 0,38. El MIDES, por su parte, destaca que "el aumento del ingreso del $20 \%$ más pobre fue $86 \%$, mientras que en el $20 \%$ más rico fue de $36 \%$ " (MIDES, 2014: 6). Añade que el $70 \%$ más pobre de la población, "en la distribución de los ingresos totales", aumento su participación pasando de un 40\% en 2006 a un 46\% en 2013 (MIDES, 2014: 29). Caetano (2016a: 41) señala que el 0,38 logrado está muy cerca del índice de los países del sur de Europa (0,34). De Armas (2017: 350-1) apunta que "Uruguay forma parte del $10 \%$ de países del mundo (a partir de un universo de ochenta países con información disponible en las bases en línea del Banco Mundial) donde más disminuyó el Coeficiente de GINI entre los quinquenios 2005-2009 y 2010-2014".

Otros/as autores/as, sin embargo, utilizando otras metodologías de medición de la desigualdad niegan que se haya producido el descenso que las cifras oficiales presentan. Notaro, por ejemplo, advierte de las limitaciones de la Encuesta Continua de Hogares (ECH), referencia principal en Uruguay para construir el índice de GINI. Para el autor, el 
problema de la $\mathrm{ECH}$ es que no identifica todos los ingresos de una familia, ya que deja fuera tres categorías de ingresos: "Ias exoneraciones, es decir, ingresos que no están gravados y por lo tanto no generan recaudación"; "los ingresos generados en el país y transferidos al exterior por intereses de IEC (Inversión Extranjera de Cartera) o utilidades de IED (Inversión Extranjera Directa)"; "Ias utilidades reinvertidas o los intereses capitalizados se consideran ingresos de personas jurídicas sin tener en cuenta que sus propietarios son personas físicas". Por tanto, el ECH subestima "los ingresos del capital" y sería necesario "otro enfoque para mejorar la estimación" (Notaro, 2016b: 7)

Notaro advierte de la paradoja de que "los hogares uruguayos declaran más ingresos de capital a la DGI (Agencia de recaudación de impuestos del gobierno) que a la ECH". Los ingresos del capital del periodo 2010-13 fueron, según el autor, "10.306,9 millones de dólares promedio anual, de los cuales 8.275 .7 son capital nacional, que son más de 13 veces superiores a los captados por la ECH en el mismo período, lo que cuestiona los índices de GINI calculados con información de esa fuente" (Notaro, 2016b: 16).

Notaro aporta otro dato que refuta el descenso de la desigualdad y es que la participación de las rentas de trabajo en el PIB ha descendido del 27\% en 1998 al 23\% en 2010 (Notaro, 2015: 19). Mañan, en la misma línea, afirma que "el salario creció en términos reales en el periodo", pero su peso en el conjunto de la riqueza generada sigue cayendo. Agrega que la redistribución (o reducción de desigualdad) se dio pero fue "al interior de la clase trabajadora, de los trabajadores de mayor salario hacia los de menor" (Mañan, 2013: 45-6). Narbondo (2014) ofrece datos del ámbito rural y asevera que "ha ocurrido un proceso concentrador. Si bien los salarios rurales han crecido en términos absolutos su peso relativo en el Valor Agregado Bruto (VAB) agropecuario cayó de $30 \%$ a $17 \%$ del año 2000 al 2013. Considerando en conjunto los ingresos de asalariados y productores familiares, estos pasaron de un $40 \%$ a un $24 \%$ del $\mathrm{VAB}$, cuando los ingresos del capital pasaron de $40 \%$ a $53 \%$ y los ingresos de los terratenientes pasaron de $8 \%$ a 16\%".

\section{Política económica}

Para evaluar la política económica de los gobiernos del FA vamos a tener en cuenta los siguientes aspectos: las medidas en relación a la matriz productiva, la evolución de la propiedad en términos de mayor o menor concentración de esta, los avances o retrocesos en clave de soberanía nacional, los cambios en el ámbito tributario y el modelo de integración con el exterior (América Latina y el resto del mundo). 
Antes de iniciar el balance resulta conveniente presentar una serie de datos relacionados con el crecimiento económico, ya que durante la primera década del FA se han dado unas tasas de crecimiento muy alto, "claramente superiores a las históricas" (Bertola y Bertoni, 2014: 66).

Notaro (2015: 9) señala que de 2004 a 2014 el PIB crecio a una tasa media anual de 5,4\%. La razón fundamental para explicar el alto crecimiento, según la mayoría de los/as expertos/as es el boom de las commoditties (Pérez, Piñeiro y Rosenblatt, 2016: 3; Caetano, 2016a: 35; REDIU, 2010: 21): "Esto permitió que Uruguay, un país exportador de alimentos y materias primas, creciera durante una década a tasas inusuales para su promedio histórico" (Pérez, Piñeiro y Rosenblatt, 2016: 3).

El citado alto precio de las materias primas nos sirve para analizar la política del gobierno en relación a la matriz productiva del país. Resulta conveniente recordar que "es una constante histórica el hecho de que Uruguay tiene sus exportaciones concentradas en una cantidad pequeña de productos" primarios: "Ios bienes primarios y manufacturas basadas en recursos naturales explican en promedio alrededor de $2 / 3$ del valor de dichas exportaciones". Se produjo "una reducción de esa participación entre 1938 y 1980", pero en la actualidad la tendencia se revierte ubicándose "en torno al 75\%", lo cual evidencia una "reprimarización" (Bertola y Bertoni, 2014: 67-8). Tanto autores favorables al gobierno como críticos coinciden en señalar este hecho (Blixen, 2017; Zibechi, 2017; Olesker, 2017; Gudynas, 2017; Abelando, 2017; Delacoste, 2017; Caetano, 2017).

La reprimarización de la última etapa se explica por dos razones. En primer lugar, en la década del noventa "la liberalización comercial hizo que muchas industrias fueran desmanteladas", en el marco de la imposición del modelo neoliberal. Durante la gestión del FA, "el contexto internacional no ha contribuido a fortalecer la postura de aquellos que promueven cambios profundos de la matriz productiva. Por el contrario, el país ha visto profundizar su dependencia de los recursos naturales, aprovechando un aumento pronunciado de la demanda y precios de estos productos" (Bertola y Bertoni, 2014: 71).

La reprimarización la explica en gran medida el crecimiento de algunos rubros, principalmente la soja y el sector forestal. La soja aumenta en 15 años su producción de manera exponencial, pasando de 10.000 hectáreas en el año 2000 a 859.000 en el 2010 (Zurbriggen, 2016: 331) y a 1.300.000 en 2014. El sector forestal pasa de ocupar 190.000 hectáreas en 1990 a casi un millón en 2013 (Narbondo, 2014).

Si tomamos como referencia el total de exportaciones se percibe un aumento de los productos primarios que pasan de representar el $20 \%$ al 
$35 \%$ del total "y la evolución inversa de la industria manufacturera que perdió un 17\% del total" (Notaro, 2015: 13). Entre el año 2001 y 2013 los ingresos generados por la soja subieron exponencialmente, pasando de un anecdótico millón y medio de dólares a 1.875 millones. La carne bovina, sector más tradicional, aumentó también de 217 a 1.298 millones de dólares. El sector forestal también creció mucho al pasar de 84 a 337 millones (Zurbriggen, 2016: 331). Por tanto, es innegable la consolidación del modelo extractivista exportador (Elias, 2010: 71; REDIU, 2010: 21; Mañan, 2013: 48).

Otro ámbito fundamental para evaluar la política económica de un gobierno es su proyecto y sus medidas en relación a la democratización de la propiedad de los medios de producción. En las "Bases Programáticas" presentadas por el Frente Amplio para el periodo de gobierno 2015-2020 no se plantea explícitamente el debate sobre la redistribución de la propiedad pero sí se promete avanzar en la Economía Social y Solidaria y específicamente "aumentar su peso en la economía" (Frente Amplio, 2014: 56).

El balance en términos de redistribución de la propiedad de la mayoría de las/os autores/as es muy crítico. Hay consenso en que se ha dado un proceso de concentración de propiedad en el campo (Zurbriggen, 2016: 331; Santos et al, 2013: 23; Mañan, 2013: 45; Blixen, 2017; Notaro, 2017; Zabalza, 2017). Mañan (2013: 49) indica que no se tomaron medidas "para romper la lógica de concentración y exclusión que se criticaba desde la misma izquierda cuando ésta era oposición".

Narbondo (2014) aporta datos del proceso de concentración de propiedad en el ámbito rural: "Los datos del último Censo General Agropecuario arrojan que entre 2000 y 2011 desaparecieron alrededor de 12.000 explotaciones agropecuarias, en su inmensa mayoría productores familiares, consolidando una estructura agraria sumamente concentrada en la que las explotaciones de más de 1.000 hectáreas, siendo el 9\% del total, controlan el 60\% de la tierra". Notaro (2015: 22) especifica que este tipo de grandes explotaciones "en la última década pasaron de controlar del $55 \%$ al $60,6 \%$ del total de la superficie productiva del país". Narbondo (2014) agrega que "asociado a este proceso ocurrió un fenómeno de creciente anonimato en la propiedad de la tierra donde entre 2000 y 2011 las personas jurídicas (sociedades anónimas en su mayoría) pasaron de controlar de un $1 \%$ a un $43,1 \%$ del territorio, apropiándose de casi 7 millones de hectáreas".

El debate sobre la propiedad tiene también su expresión en relación a la soberanía nacional, es decir, a la necesidad de evaluar la evolución del control de los medios de producción por parte de nacionales o extranjeros. Todas las fuentes consultadas coinciden en que se ha producido en estos últimos años un proceso de "extranjerización" de los 
sectores más dinámicos de la economía (agrario y forestal). (Blixen, 2017; Olesker, 2017; Delacoste, 2017; Rubio, 2017).

La extranjerización de la tierra es el fenómeno más destacado por la mayoría de investigadores/as. Mañan (2013: 48) indica que la expansión "del complejo forestal-pastero" ha generado "un desplazamiento de otros complejos tradicionales de capital nacional". Santos et al (2013: 22) sostienen que en relación al "control de la tierra entre 2000 y 2011 los propietarios uruguayos pasaron de $90 \%$ a $54 \%$ en el control de la superficie nacional". Notaro (2015: 22) subraya que en cuanto a la superficie agraria "27 grupos transnacionales concentran 1.640 .000 hectáreas, casí la misma superficie que los 21.645 establecimientos registrados como agricultores familiares". Según el mismo autor, hoy día un tercio de la superficie agropecuaria está extranjerizada (Notaro, 2016a: 482). Hay sectores agrarios donde el nivel de extranjerización es muy alto, como por ejemplo el arroz, donde el $87 \%$ de la industria son capitales brasileños (Notaro, 2015: 22). En el área forestal la concentración y extranjerización se combinan: "8 empresas son propietarias de $72 \%$ de las tierras forestadas y 4 son transnacionales (Notaro, 2016a: 482). El proceso de extranjerización se expresa también en la dinámica exportadora. Santos et al (2013: 22) aseguran que en 2012 "de las diez principales empresas exportadoras, ocho fueron extranjeras".

Todo este proceso de pérdida de soberanía económica está relacionado con el alto nivel de inversión a través de la Inversión Extranjera Directa (IED). Indudablemente la IED ha dinamizado la economía y ha sido un motor fundamental del crecimiento económico, pero a su vez no se pueden negar sus consecuencias en términos de extranjerización de la economía. Caetano (2016a: 49) destaca que la IED ha sido la "más alta del último medio siglo": si durante la década del 90 significó entre un 12 y un $17 \%$ del PIB entre 2005 y 2015 se movió en una horquilla del 17 al $23 \%$ del PIB. Notaro (2015: 14) recuerda que "el ingreso anual de IED en Uruguay era el 2.8\% del PIB en 2003-2004 y llegó a $5.0 \%$ en 2012-2014, por lo que el país se convirtió en uno de los mayores receptores de flujos de inversión en términos relativos de la región".

Otro aspecto central para analizar el cambio económico es la dimensión ecológica. Narbondo (2014) asegura que el modelo ha provocado impactos evidentes en el suelo, el agua y la biodiversidad. "En el caso de los suelos el proceso de intensificación de la agricultura supuso un incremento sustancial de los riesgos de erosión y degradación como resultado de al menos tres aspectos: la sustitución de las rotaciones de cultivos con pasturas por sistemas de agricultura continua, el incremento de la frecuencia de la soja en las rotaciones, y la expansión de la agricultura hacia zonas no tradicionales con suelos con 
mayor riesgo de degradación". Respecto al agua, "en los últimos años se ha registrado contaminación por elevadas cargas de nutrientes en distintas cuencas, lo que estaría asociado a un modelo tecnológico que provoca altos niveles de erosión".

Notaro (2015: 33) menciona expresamente "el efecto de la soja en la pérdida de fertilidad de la tierra" y el problema de la "toxicidad" provocada por la fumigación. Zurbriggen (2016: 332) resalta concretamente la contaminación de alimentos debido al uso creciente de "plaguicidas u otros productos químicos". Gudynas (2017) asegura que "han desaparecido una buena parte de los humedales por la expansión del arroz".

El modelo de relaciones comerciales internacionales es otro ámbito fundamental para evaluar. Aquí vamos a analizar dos aspectos: por una parte, la evolución de la integración económica regional latinoamericana y concretamente con los países del MERCOSUR; y por otra parte, el posicionamiento ante la liberalización comercial internacional. Respecto a la primera cuestión, en las "Bases Programáticas" presentadas por el Frente Amplio para el periodo de gobierno 2015-2020 se realiza una apuesta expresa por fortalecer la integración regional: "La inserción del Uruguay en el mundo será impulsada desde la plataforma MERCOSUR. Por ello se fomentará una agenda externa común del bloque, consolidando y fortaleciendo las Instituciones Regionales" (Frente Amplio, 2014: 140).

En la práctica, sin embargo, las nuevas tendencias de la economía internacional, principalmente la gran demanda china, operaron objetivamente en contra de la ruta integracionista latinoamericana: "Ia economía internacional ofreció señales poco propicias para la refundación del bloque. El boom de los precios de los commodities reforzó la especialización primario exportadora y gestó una suerte de relación neoperiférica con China" (Bertola y Bertoni, 2014: 92).

En 1998 se exportaba a China apenas 76 millones de dólares, mientras que en 2014 el monto era de 1.220 millones. Es relevante la caída de los dos principales mercados de exportación (Brasil y Argentina). Brasil pasa de recibir el 33,8\% de todas las exportaciones en 1998 a un $18 \%$ en 2014. Argentina baja del $18,5 \%$ al $4,9 \%$. Al contrario, China sube exponencialmente hasta acaparar el 22,5\% del total en 2014. (Notaro, 2015: 12-14). China se convierte por tanto en el principal destino de las exportaciones, mientras que el MERCOSUR, "que representaba un 51,5\% de sus exportaciones en 1998, representa solo un $26 \%$ en 2013" (Zurbriggen, 2016: 330). A su vez, "China pasó al primer lugar como proveedor con un $18.5 \%$ del total de las importaciones, desplazando a Argentina y a Brasil". Brasil cae del $20,8 \%$ al $17 \%$ y Argentina del $22 \%$ al 12,7\% (Notaro, 2015: 12-14). 
En cuanto al posicionamiento del gobierno ante la liberalización comercial habría que evaluar dos conflictos recientes. En primer lugar, las diferencias" dentro del FA en relación a la "presencia del país en las negociaciones del TISA", es decir, en el intento de acuerdo internacional para liberalizar los servicios. Asegura Caetano que el gobierno de Mujica participó de manera "secreta", sin conocimiento de las bases del FA, lo cual "generó una fuerte polémica en el seno de la izquierda al cobrar publicidad en los primeros meses del gobierno de Tabaré Vázquez". Vázquez "resolvió pasarlo a decisión del Plenario nacional del Frente Amplio, el que por una amplísima mayoría lo rechazó y exigió el retiro inmediato de las negociaciones. El Presidente Vázquez cumplió de inmediato con esa resolución de las autoridades frenteamplistas" (Caetano, 2016b). En segundo lugar, el intento de firmar un TLC con Chile, que de nuevo fue frenado por las bases del FA, que se opusieron el gobierno (Canzani, 2018).

El último ámbito que queremos analizar es el sistema tributario. La mayoría de las reformas se pusieron en marcha durante el primer gobierno de Tabaré Vázquez (2005-2009) y fueron las siguientes: "(i) la creación del Impuesto a la Renta de las Personas Físicas (IRPF), que sustituyó al anterior Impuesto a las Retribuciones Personales (IRP)"; (ii) la "creación del Impuesto a la Renta Empresarial (IRAE), que sustituyó al Impuesto a las Rentas de la Industria y Comercio (IRIC); (iii) la disminución del peso de la tributación indirecta". Además también se creó el "Impuesto a la Renta de las Personas no Residentes (IRNR), que grava las utilidades o dividendos percibidos por no residentes, con tasas similares a las rentas del capital del IRPF". Finalmente, también se impulsaron algunas reformas en relación a la transparencia financiera (Antia, 2016: 304-5).

La creación del IRPF es la reforma tributaria más relevante del Frente Amplio. Las novedades que introduce el nuevo impuesto son las siguientes: ahora "las rentas del capital y las que perciben los profesionales, que hasta entonces habían sido exentas, pasaron a estar gravadas"; además, se exonera del impuesto a la población de bajos ingresos (alrededor de un 60\%); finalmente, "permite muy pocas deducciones" (Antia, 2016: 305).

Respecto a la valoración de la reforma nos encontramos con perspectivas diversas. Por un lado, algunos/as autores/as subrayan que la reforma del IRPF se caracteriza por ser de carácter "progresivo" y por tanto haber avanzado en términos de equidad (Antia, 2016: 305; De Armas, 2017: 350; Olesker, 2014a:22; Pérez, Piñeiro y Rosenblatt, 2016: 6; Hagobian, 2017; Mahia, 2017; Lazo, 2017; Tajam, 2017; Agassi, 2017). Betancur y Busquets (2016: 385) destacan que la reforma permitió un "notorio incremento" de los ingresos para el gobierno. 
Bertola y Bertoni (2014: 83), sin embargo, consideran que la progresividad fijada en el nuevo IRPF es tímida. Mañan (2013: 42) y REDIU (REDIU, 2010: 16) precisan que la reforma es "progresiva" respecto a las rentas de trabajo pero no respecto a las rentas de capital, que son gravadas con una "tasa plana". Para Mañan (2013: 43) la redistribución se da entre los y las asalariadas, ya que la exoneración fiscal a los sectores de ingresos más bajos (60-70\%) se compensa "con cargo al 10\% de la masa salarial que financió la descompresión fiscal". REDIU (2010: 17) sostiene que la mayoría de la recaudación del IRPF viene de las clases trabajadoras: "si se atiende a la recaudación total del nuevo impuesto (IRPF), esta depende en un $87 \%$ de las rentas del trabajo y solo un 13\% de las rentas del capital". De todas formas, Mañan (2013: 43) si rescata tres aspectos positivos de la reforma: que los sectores profesionales antes exentos hoy contribuyen; al igual "que los depósitos bancarios de los residentes"; y que "los ingresos por alquileres y las transacciones inmobiliarias" son también gravados.

La reforma al impuesto empresarial genera un alto nivel de rechazo ya que implicó una reducción tributaria a la patronal. Con el antiguo IRIC las empresas pagaban un $30 \%$, mientras que con el nuevo IRAE pasan a pagar un 25\% (Mañan, 2013: 42; Santos et al, 2013: 17). Antia (2016: 305) indica que esta caída intentó compensarse con la siguiente medida: "se estableció que los dividendos distribuidos pagarían un 7 \% adicional, mediante el IRPF a las rentas del capital, con lo cual para las empresas que distribuyeran utilidades la tasa quedó situada a un nivel similar a la que regía en el período prerreforma". Otro retroceso en términos tributarios sería la reducción de tasas del Impuesto al Patrimonio (Notaro, 2017).

La disminución del peso de la tributación indirecta se tradujo en la bajada del IVA de los productos de la canasta básica, del 14\% al 10\% (Olesker, 2014a:22). Las reformas en relación a la transparencia financiera son calificadas por Antía como moderadas: "Ello incluyó una modesta flexibilización del secreto bancario, la prohibición de crear nuevas Sociedades Anónimas Financieras de Inversión (SAFI) y el establecimiento de un plazo para la desaparición de las SAFI existentes. La nueva política en materia de SAFI tuvo el propósito de evitar que el país fuera considerado un paraíso fiscal" (Antia, 2016: 305).

Las reformas tributarias del gobierno de Mujica (2010-15) se centraron en dos áreas: reforma de distintas normas relativas a la "transparencia, el régimen de secreto bancario y de intercambio de información tributaria con otros países", y el intento de "creación de un nuevo tributo para desalentar la concentración de la tierra en el medio rural" (Antia, 2016: 305). En relación a la primera área, el Ejecutivo de Mujica impulsó una reforma "moderada" para flexibilizar el secreto bancario y firmó 
acuerdos para intercambiar "información tributaria con otros países" (Antia, 2016: 305).

A su vez, Mujica intentó implantar un tributo "para gravar a los grandes propietarios rurales" a través de la creación del Impuesto a la Concentración de Inmuebles Rurales" (ICIR), que pretendía "desalentar la concentración de la tierra". El tributo estaba dirigido al 2,5\% de los terratenientes más grandes, "quienes poseen un $36 \%$ de la superficie total de tierra". Los sectores afectados agrupados en dos patronales poderosas, la "Asociación Rural del Uruguay (ARU) y la Federación Rural del Uruguay (FRU) criticaron enérgicamente el proyecto de ley" y lograron que la la Suprema Corte de Justicia (SCJ) invalidara el impuesto (Antia, 2016: 308-10).

En el balance global en torno a las reformas tributarias hay que valorar el incremento de ingresos vía IRPF pero también la modesta tributación del capital. Bertola y Bertoni (2014: 83) resaltan que el modelo tributario no ha logrado "captar ingresos de los beneficios extraordinarios provenientes de la acumulación de activos o de ganancias originadas en coyunturas de precios muy favorables". Incluso los autores más favorables al gobierno reconocen que el gran capital no ha sido gravado lo suficiente (Olesker, 2017; Caetano, 2017).

El balance global de la economía, obviamente no es tan positivo como el de las políticas sociales. Algunos/as investigadores/as caracterizan al modelo como neodesarrollista (Santos eta al, 2013; Gudynas, 2017; Zibechi, 2017; Notaro, 2017). El neodesarrollismo "significa un cambio respecto del modelo neoliberal" por el papel más interventor del Estado y por las mejoras de las condiciones sociales de las clases populares, pero profundiza el "régimen de acumulación favorable al capital transnacional", con la "llegada masiva de IED" y el otorgamiento de privilegios legales a las empresas extranjeras. Se ha consolidado el carácter primario de la economía y se ha intensificado "el proceso de extranjerización de la propiedad y de la producción" (Santos et al, 2013: 13-22).

\section{Conclusiones}

La victoria electoral del Frente Amplio en Uruguay y su experiencia de gobierno hasta el momento han supuesto un punto de inflexión en la historia política del país, no solo porque los partidos tradicionales han sido desplazados del poder Ejecutivo tras más de un siglo, sino porque el proyecto político del FA ha traído un cambio respecto al modelo dominante que se empezó a imponer en la dictadura y se consolidó en la década de los noventa del siglo pasado. Teniendo claro, por tanto, que se ha producido un cambio, resulta casi más importante 
caracterizarlo, dimensionando su profundidad. Esto nos exige alejarnos de las lecturas extremistas de algunas y algunos analistas, que dejándose llevar por lealtades políticas y/o por dogmatismos ideológicos, dibujan un panorama más cercano a sus esquemas mentales que a la realidad. Los análisis complacientes con el cambio que llegan incluso a considerar el proceso uruguayo como uno de los más avanzados de los ensayos post-neoliberales en América Latina, resultan desconcertantes y hacen un flaco favor a cualquier agenda de izquierda realmente rupturista. De la misma manera, las evaluaciones catastróficas que proyectan una imagen del país estática, como si nada hubiera cambiado, no solo niegan la realidad sino que restan su justo valor a la mejora de las condiciones de vida de las clases populares. El cambio en Uruguay es reformista, es moderado, es tibio, ni más, ni menos.

Sin duda alguna, el área social es donde el cambio ha tenido su principal reflejo, a través de un nuevo enfoque de políticas sociales, en las que se han desterrado algunos de los pilares del anterior modelo neoliberal (desinversión social, privatización progresiva, mercantilización creciente, segmentación acelerada...), por nuevos principios, como el aumento sustancial del gasto social, la apuesta por recuperar la universalización, el intento de reducir la dualización... A su vez, los indicadores más importantes han mejorado de manera evidente e incontestable, evidenciando una mejora de las condiciones materiales de los sectores más vulnerables y de las clases trabajadoras en general. La reducción drástica de la pobreza y la indigencia, el aumento de la cobertura de atención sanitaria, la mejora del poder adquisitivo de la mayoría de la población gracias al sostenido incremento del salario mínimo y del salario medio, son algunos de los indicadores que muestran que las políticas gubernamentales han dado frutos positivos y tangibles. El reto se centra ahora no solo en mejorar estas condiciones sino en hacerlas sostenibles en el tiempo, sobre todo en el actual momento de desaceleración económica que augura escenarios de mayor conflictividad.

El área económica es donde los análisis más complacientes no tienen mucha credibilidad. En primer lugar, hay que recordar que desde el primer día del primer gobierno del FA el ministerio de Economía ha estado bajo control del sector más liberal del Frente Amplio, el denominado Frente Liber Seregni. Esto fue una señal para tranquilizar a los poderes fácticos y para intentar así lograr un clima de cierta paz social con el capital, tanto nacional como internacional. Indudablemente esta táctica ha tenido éxito en ese sentido, ya que el Ejecutivo frenteamplista no ha tenido que soportar la agresión y en algunos casos desestabilización que otros gobiernos de la región han padecido. Sin embargo, esta calma política ha tenido como contraparte que la agenda económica haya sido sumamente 
conservadora en términos de construir un nuevo modelo. Algunos indicadores económicos han sido exitosos (crecimiento económico, mayor IED, etc.) en un contexto global favorable, pero hay que tener claro que no son referentes para evaluar cambios en clave de izquierda. Los horizontes y orientaciones de un proyecto de izquierda rupturista, como la desconcentración de la propiedad o el avance en soberanía económica, entre otros, no han estado apenas presentes en la política económica. Paralelamente, el aumento de la primarización de la economía evidencia que el neodesarrollismo es un rasgo característico del actual Ejecutivo. Esta última obviedad no oculta que criticar el neodesarrollismo es mucho más sencillo que superarlo en la práctica, en un sistema-mundo en el que un país pequeño del Sur tiene condicionantes estructurales poco favorables.

A principios de este siglo XXI, y tras casi 3 lustros en el gobierno, se puede afirmar que el Frente Amplio ha demostrado una gran astucia y habilidad para mantenerse en el Ejecutivo, en gran medida por su capacidad de adaptarse a la realidad sociológica del país. Esto se puede interpretar como una virtud, pero a su vez ejerce como un freno para posibles cambios más profundos. El FA, como algunos/as expertos/as plantean, es hoy día, con lo bueno y con lo malo que eso implica, la socialdemocracia ( $\grave{\circ}$ el social-liberalismo?) de la política uruguaya, o como diría Arocena, el 'batllismo del siglo XXI'. En momentos de crecimiento económico y viento favorable el proyecto ha funcionado, pero en la actual coyuntura de recesión y de mayor conflictividad, quedarse en el centro será cada vez más difícil.

\section{Bibliografía}

ANTIA, Florencia et al (2013). "La renovación del sistema de protección uruguayo: el desafío de superar la dualización". Revista urúguaya de Ciencia Política, vol. 22, n², pp. 171-194.

ANTIA, Florencia (2016). "La política tributaria durante el gobierno de Mujica: zhacia el fin del secreto bancario?", en Betancur, N. y Busquets, J.M. El decenio progresista. Las políticas públicas de Vázquez a Mujica. Fin de Siglo. Montevideo, pp. 301-326.

AROCENA, Rodrigo (2005). "Uruguay en la nueva ola de las izquierdas latinoamericanas". Nueva Sociedad, n 197, mayo-junio, pp. 146-158.

BERTOLA, LUis; BERTONI, Reto (2014). "Sinuosa y convulsa: la economía uruguaya en el último medio siglo", en Harari et al. Uruguay + 25. Fundación Astur, pp. 65-94.

BETANCUR, Nicolás; BUSQUETS, José Miguel (2016). "Una mirada de conjunto a las políticas públicas del decenio progresista", en Betancur, 
N. y Busquets, J.M. El decenio progresista. Las políticas públicas de Vázquez a Mujica. Fin de Siglo. Montevideo, pp. 373-393.

CAETANO, Gerardo (2016a). "Balances y desafíos en Uruguay, luego de una década de gobierno de la izquierda". Cadernos Prolam/USP 15 (28), pp. 32-52.

CAETANO, Gerardo (2016b). "Balances provisorios del primer año de la tercera administración del Frente Amplio: ¿ffreno o impulso?". Nueva Sociedad, marzo 2016

http://nuso.org/articulo/balances-provisorios-del-primer-ano-de-la-

tercera-administracion-del-frente-amplio-freno-o-impulso/

CANZANI, Agustín (2018). "El Frente Amplio en la encrucijada. Debates, tensiones y matices en la izquierda uruguaya". Nueva Sociedad. Mayo. http://nuso.org/articulo/el-frente-amplio-en-la-encrucijada/

CARRACEDO, Fabián; SENATORE, Luis (2016). "Las políticas laborales y las relaciones de trabajo durante el gobierno de José Mujica", en Betancur, N. y Busquets, J.M. El decenio progresista. Las políticas públicas de Vázquez a Mujica. Fin de Siglo. Montevideo, pp. 15-41.

DE ARMAS, Gustavo (2017). Reformas sociales y gobiernos de izquierda en Uruguay (2005-2014).

Tesis Doctoral. Universidad de la República. Uruguay.

ELIAS, Antonio (2010). "La izquierda progresista y el proyecto del capital", en REDIU. La torta y las migajas. Trilce. Montevideo, pp. 71-85.

FRENTE AMPLIO (2014). Bases programáticas. Tercer gobierno nacional del Frente Amplio 2015-2020. Comisión Nacional del Frente Amplio. Montevideo

LORENZONI, Miguel; PÉREZ, Verónica (2013). "Cambios y continuidades de la izquierda en Uruguay: un análisis a partir de las propuestas programáticas del Frente Amplio 1971-2009". Revista Uruguaya de Ciencia Política, vol. 22, n 1. Montevideo, pp. 81-102.

MAÑAN, Oscar (2013). "Uruguay y la era progresista: gatopardismo y desencanto". Comercio Exterior, Vol. 63, n 6, pp. 41-49.

MIDAGLIA, Carmen (2018). "La izquierda política y la reforma social en el Uruguay del siglo XXI". Ponencia presentada en el $56^{\circ}$ Congreso de Americanistas. Salamanca. 15-20 julio.

MINISTERIO DE DESARROLLO SOCIAL (MIDES) (2014). Revisión de indicadores básicos de desarrollo social 2006-2013. MIDES.

NARBONDO, Ignacio (2014). "El agro en Uruguay: los cambios detrás de los cambios". Brecha, 1518, diciembre. Montevideo.

NOTARO, Jorge (2015). "La economía del Uruguay 1998-2014. Caracterización e hipótesis explicativas". Manuscrito facilitado por el autor. Montevideo.

NOTARO, Jorge (2016a). "Uruguay 2015: la opción por el ajuste". Rebela, Revista Brasilera de Estudos Latino-Americanos, vol. 6, n 3, pp. 459-497. NOTARO, Jorge (2016b). "Los ingresos del capital. Uruguay 2008-2013". Researchgate.net.

https://www.researchgate.net/publication/311398188_LOS_INGRESOS_D EL_CAPITAL_URUGUAY_2008_-_2013 
OLESKER, Daniel (2014a). "Nueve años de gobierno frentamplista". http://www.chasque.net/vecinet/DOlesker.pdf

OLESKER, Daniel (2014b). "El modelo de desarrollo desde una perspectiva de América Latina: el caso uruguayo". Manuscrito facilitado por el autor. Montevideo

PÉREZ, Verónica y PIÑEIRO, Rafael (2016). "Uruguay 2015: los desafíos de gobernar por izquierda cuando la economía se contrae". Revista de Ciencia Política, vol. 36, n¹. Santiago de Chile, pp-339-363.

PÉREZ, Verónica; PIÑEIRO, Rafael; ROSENBLATT, Fernando (2016). "El Frente Amplio tras once años en el gobierno". Friedricht Ebert, Uruguay, $\mathrm{n}^{\circ} 18$

REDIU (2010). La torta y las migajas. Trilce. Montevideo.

SANTOS, Carlos et al (2013). "Seis tesis sobre el neodesarrollismo en Uruguay". Contrapunto, n². Montevideo, pp. 13-32.

ZURBRIGGEN, Cristina (2016). "Uruguay, un país 'agro inteligente'", en Betancur, N. y Busquets, J.M. El decenio progresista. Las políticas públicas de Vázquez a Mujica. Fin de Siglo. Montevideo, pp. 301-326.

\section{Entrevistas}

ABELANDO, Víctor Hugo (2017). Entrevista personal. 18-04-2017. AGASSI, Ernesto (2017). Entrevista personal. 29-04-2017.

BLIXEN, Samuel (2017). Entrevista personal. 07-04-2017. CAETANO, Gerardo (2017). Entrevista personal. 11-04-2017. DELACOSTE, Gabriel (2017). Entrevista personal. 20-04-2017. GUDYNAS, Eduardo (2017). Entrevista personal. 14-04-2017. HAGOBIAN, Sebastián (2017). Entrevista personal. 19-04-2017. LAZO, Sandra (2017). Entrevista personal. 26-04-2017. LORIER, Eduardo (2017). Entrevista personal. 28-04-2017. MAHIA, José Carlos (2017). Entrevista personal. 25-04-2017. NOTARO, Jorge (2017). Entrevista personal. 15-04-2017. OLESKER, Daniel (2017). Entrevista personal. 10-04-2017. PEREIRA, Marcelo (2017). Entrevista personal. 19-04-2107. RUBIO, Eduardo (2017). Entrevista personal. 25-04-2017. TAJAM, Hector (2017). Entrevista personal. 26-04-2107. ZABALZA, Jorge (2017). Entrevista personal. 17-04-2017. ZIBECHI, Raúl (2017). Entrevista personal. 07-04-2017. 